\title{
Non-Bloch quench dynamics
}

\author{
Tianyu Li $\odot,{ }^{1}$ Jia-Zheng Sun, ${ }^{1}$ Yong-Sheng Zhang, ${ }^{1,2, *}$ and Wei $\mathrm{Yi}^{1,2, \dagger}$ \\ ${ }^{1}$ CAS Key Laboratory of Quantum Information, University of Science and Technology of China, Hefei 230026, China \\ ${ }^{2}$ CAS Center for Excellence in Quantum Information and Quantum Physics, Hefei 230026, China
}

(Received 24 January 2021; revised 22 March 2021; accepted 23 March 2021; published 7 April 2021)

\begin{abstract}
We study the quench dynamics of non-Hermitian topological models with non-Hermitian skin effects. Adopting the non-Bloch band theory and projecting quench dynamics onto the generalized Brillouin zone, we find that emergent topological structures, in the form of dynamic skyrmions, exist in the generalized momentumtime domain, and are correlated with the non-Bloch topological invariants of the static Hamiltonians. The skyrmion structures anchor on the fixed points of dynamics whose existence is conditional on the coincidence of generalized Brillouin zones of the pre- and post-quench Hamiltonians. Global features of dynamic skyrmions, however, persist well beyond such a condition, constituting signatures for a general dynamic detection scheme of non-Bloch topology in the presence of non-Hermitian skin effects. Applying our theory to an experimentally relevant, nonunitary quantum walk, we explicitly demonstrate how the non-Bloch topological invariants can be revealed through the non-Bloch quench dynamics.
\end{abstract}

DOI: 10.1103/PhysRevResearch.3.023022

\section{INTRODUCTION}

Non-Hermitian Hamiltonians arise in open systems [1,2], and have attracted significant attention [3-10]. As a peculiar feature of a wide class of non-Hermitian Hamiltonians, their nominal bulk eigenstates are localized near boundaries under what is now known as the non-Hermitian skin effects [11-23]. Remarkably, for non-Hermitian topological models with skin effects, the conventional bulk-boundary correspondence breaks down [11-18,24], whose restoration calls for a non-Bloch band theory where topological invariants are calculated in the generalized Brillouin zone (GBZ) under relevant boundary conditions [11-15,23,25,26], rather than in the conventional Brillouin zone (BZ) under a periodic boundary condition (PBC). Both non-Hermitian skin effects and non-Bloch bulk-boundary correspondence have recently been experimentally confirmed [27-32]. However, a direct detection of non-Bloch topological invariants is yet to be demonstrated. Here we show how non-Bloch topological invariants can be determined through dynamic topological structures in quantum quenches that are experimentally accessible.

A quantum quench describes the evolution of an eigenstate of the initial Hamiltonian $H^{i}$ driven by a final Hamiltonian $H^{f}$. Particularly, for one-dimensional topological systems, previous studies have shown that dynamic skyrmions, a topo-

\footnotetext{
*yshzhang@ustc.edu.cn

†wyiz@ustc.edu.cn
}

Published by the American Physical Society under the terms of the Creative Commons Attribution 4.0 International license. Further distribution of this work must maintain attribution to the author(s) and the published article's title, journal citation, and DOI. logical structure hinged upon fixed points of dynamics, should appear in the emergent momentum-time domain, when $H^{i}$ and $H^{f}$ possess distinct topological properties [33-35]. Hence, by studying the dynamic signatures of a quantum quench, one gains information regarding the topological invariants of the pre- and post-quench Hamiltonians [36-55]. Similar conclusions hold for nonunitary quenches governed by non-Hermitian Hamiltonians, but are predicated upon two conditions [35]: (i) the decoupling of dynamics in different momentum sectors under the lattice translational symmetry; and (ii) reality of the eigenenergy spectra of both $H^{i}$ and $H^{f}$, where the parity-time $(\mathcal{P} T)$ symmetry plays an important role [2-6]. For a non-Hermitian topological model with skin effects, however, a compromise between these two requirements seems to be a tall order. On one hand, dynamics of different momentum states are no longer decoupled under an open boundary condition (OBC). On the other, for a system possessing non-Hermitian skin effects, the associated Bloch spectra in the BZ necessarily form loops in the complex plane [21,22], such that real eigenspectra only exist in the GBZ under OBCs, protected by a non-Bloch $\mathcal{P} T$ symmetry $[32,56,57]$.

We circumvent these issues by projecting the quench dynamics onto the generalized momentum sectors of the GBZ. Such a non-Bloch analysis enables us to reveal, in the generalized momentum-time domain, dynamic skyrmions that are intimately related to the non-Bloch topological invariants of the pre- and post-quench Hamiltonians. In particular, for a non-Hermitian Su-Schrieffer-Heeger (SSH) model with asymmetric hopping and under $\mathrm{OBC}$, we prove that the existence of the underpinning fixed points for dynamic skyrmions exist, provided $H^{i}$ and $H^{f}$ both have real eigenspectra and feature the same GBZ, but with distinct non-Bloch winding numbers. When the GBZs of $H^{i}$ and $H^{f}$ are different, fixed points only exist in a perturbative sense, whereas the 
global signatures of dynamic skyrmions persist, enabling the detection of non-Bloch winding numbers. We illustrate the extraction of non-Bloch winding numbers from the non-Bloch quench dynamics, using the recently implemented, nonunitary topological quantum walk.

\section{QUENCHING IN THE GBZ}

We first consider the quench dynamics of a non-Hermitian SSH model [11,58]

$$
\begin{aligned}
H= & \sum_{n}\left[\left(t_{1}+\gamma\right)|n, A\rangle\left\langle n, B\left|+\left(t_{1}-\gamma\right)\right| n, B\right\rangle\langle n, A|\right. \\
& \left.+t_{2}|n, B\rangle\left\langle n+1, A\left|+t_{2}\right| n+1, A\right\rangle\langle n, B|\right],
\end{aligned}
$$

where each unit cell (labeled $n$ ) has two sublattice sites (labeled $A$ and $B$ ), with hopping rates given by $t_{1} \pm \gamma$ and $t_{2}$, respectively. The asymmetric intracell hopping here gives rise to non-Hermitian skin effects, such that topological properties of the model under OBC are characterized by the non-Bloch band theory, where a GBZ must be invoked, due to the deviation of the nominal bulk states from extended Bloch waves.

Specifically, the GBZ of Hamiltonian (1) under OBC is a circle with radius $r=\sqrt{\left|\left(t_{1}-\gamma\right) /\left(t_{1}+\gamma\right)\right|}$ on the complex plane [11,59], where we denote the azimuth angle as $k \in$ $(\pi, \pi]$. In the Hermitian limit with $\gamma=0$, the GBZ reduces to the conventional BZ, a unit circle on the complex plane, with $r=1$ and $k$ being the Bloch quasimomentum. As the nonHermitian counterparts of the Bloch states, we then introduce the biorthogonal spatial basis in a generalized momentum space characterized by $k:\left|\beta_{k, R(L)}\right\rangle=\frac{1}{\sqrt{N}} \sum_{n} r^{ \pm n} e^{i k n}|n\rangle(N$ is the number of unit cells), which satisfy [60]: $\left\langle\beta_{k, L} \mid \beta_{k^{\prime}, R}\right\rangle=$ $\delta_{k, k^{\prime}}$ and $\sum_{k}\left|\beta_{k, R}\right\rangle\left\langle\beta_{k, L}\right|=\mathbf{1}_{n}$, with $\mathbf{1}_{n}=\sum_{n}|n\rangle\langle n|$. Conveniently, while $\left|\beta_{k, R / L}\right\rangle$ become extended Bloch states in the Hermitian limit, $\left|\beta_{k, R}\right\rangle\left(\left|\beta_{k, L}\right\rangle\right)$ is localized toward the right (left) boundary for $r>1$, and vice versa, thus constituting a natural basis for both Hermitian and non-Hermitian cases.

We then define the projection operator $P_{k}=\left|\beta_{k, R}\right\rangle\left\langle\beta_{k, L}\right| \otimes$ $\mathbf{1}_{\alpha}, \mathbf{1}_{\alpha}$ being the identity operator in the sublattice basis $\{|A\rangle,|B\rangle\}$, and project Hamiltonian (1) onto the GBZ as $H_{k}=$ $P_{k} H P_{k}$. Writing $H_{k}=\sum_{i} d_{i}(k) \sigma_{i}$ ( $\sigma_{i}$ are the Pauli operators with $i=1,2,3)$, the non-Bloch winding number is

$$
v=\frac{1}{2 \pi} \int d k \frac{-d_{2} \partial_{k} d_{1}+d_{1} \partial_{k} d_{2}}{d_{1}^{2}+d_{2}^{2}},
$$

which recovers the bulk-boundary correspondence under OBC [11]. Note that while the eigenspectrum under PBC is always complex, it is completely real under OBC for $\left|t_{1}\right|>\gamma$, due to the presence of a non-Bloch $\mathcal{P} T$ symmetry [56,57]. Following Eq. (2), the non-Bloch topological phase boundaries in this $\mathcal{P} T$-unbroken region are given by $t_{2}= \pm \sqrt{t_{1}^{2}-\gamma^{2}}$, which reduce to those of a Hermitian SSH model when $\gamma=0$, when Eq. (2) yields the Bloch winding number.

For a state initialized in $\left|\psi_{k}^{i}\right\rangle$ of a given $k$ sector, the density matrix $\rho(t)$ of a quench process evolves as

$$
\rho(t)=e^{-i H^{f} t}\left|\Psi_{k}^{i}\right\rangle\left\langle\Psi_{k}^{i}\right| e^{i H^{f \dagger} t},
$$

where $\left|\Psi_{k}^{i}\right\rangle=\left|\beta_{k, R}^{i}\right\rangle \otimes\left|\psi_{k}^{i}\right\rangle$, with $\left|\beta_{k, R}^{i}\right\rangle$ the right spatial basis of $H^{i}$. Unlike the quasimomentum in the BZ, generalized momentum $k$ in a GBZ is not a good quantum number, as $\left\langle\beta_{k, L}^{f}\left|H^{f}\right| \beta_{k^{\prime}, R}^{i}\right\rangle \neq 0$ for $\gamma \neq 0$, such that a state initialized in a $k$ sector inevitably proliferates into other $k$ sectors in the time evolution. However, it can be shown that for $r_{i}=r_{f}$, i.e., the GBZs of $H^{i}$ and $H^{f}$ coincide with each other, these cross-coupling terms vanish in the thermodynamic limit, rendering the dynamics block-diagonal in the GBZ, similar to the conventional quench dynamics in the BZ.

Inspired by such an observation, we first focus on the simpler case of $r_{i}=r_{f}$, and discuss more general scenarios later. As a key element of non-Bloch quench, we project $\rho(t)$ onto the GBZ of $H^{f}$ [59]:

$$
\rho(k, t)=P_{k} \rho(t) P_{k}^{\dagger},
$$

where $P_{k}^{\dagger}$ is introduced to accommodate the time evolution of $\left\langle\Psi_{k}^{i}\right|$ in Eq. (3). We characterize dynamics in each $k$ sector with the dynamic spin texture $\boldsymbol{n}(k, t)[35,55]$ :

$$
\boldsymbol{n}(k, t)=\frac{\operatorname{Tr}[\rho(k, t) \eta \boldsymbol{\tau}]}{\operatorname{Tr}[\rho(k, t) \eta]},
$$

where $\boldsymbol{\tau}=\left(\tau_{1}, \tau_{2}, \tau_{3}\right)$, with $\tau_{i}=\sum_{\mu \nu= \pm}\left|\psi_{k, \mu}^{f}\right\rangle \sigma_{i}^{\mu \nu}\left\langle\chi_{k, v}^{f}\right|(i=$ 1, 2, 3). Here, $\sigma_{i}^{\mu \nu}$ are the elements of Pauli matrices $\sigma_{i}$, and $\sigma_{0}$ is a $2 \times 2$ identity matrix. $\left|\psi_{k, \pm}^{f}\right\rangle\left(\left\langle\chi_{k, \pm}^{f}\right|\right)$ is the right (left) eigenstate of $H_{k}^{f}$, with $H_{k}^{f}\left|\psi_{k, \pm}^{f}\right\rangle=E_{k, \pm}^{f}\left|\psi_{k, \pm}^{f}\right\rangle\left(H_{k}^{f \dagger}\left|\chi_{k, \pm}^{f}\right\rangle=\right.$ $\left.E_{k, \pm}^{f *}\left|\chi_{k, \pm}^{f}\right\rangle\right)$, and \pm are the band indices. The metric operator $\eta=\sum_{\mu}\left|\chi_{k, \mu}^{f}\right\rangle\left\langle\chi_{k, \mu}^{f}\right|$ is introduced to normalize $\rho(k, t)$, such that $\boldsymbol{n}(k, t)$ is a unit, real vector that can be visualized on the Bloch sphere $S^{2}[35,60]$.

Without loss of generality, we consider the initial state in each $k$ sector $\left|\psi_{k,-}^{i}\right\rangle$ to lie within the lower band, with $H_{k}^{i}\left|\psi_{k,-}^{i}\right\rangle=E_{k,-}^{i}\left|\psi_{k,-}^{i}\right\rangle$. Importantly, when $E_{k, \mu}^{f}$ is real, $\boldsymbol{n}(k, t)$ rotates around the north pole of the Bloch sphere with a

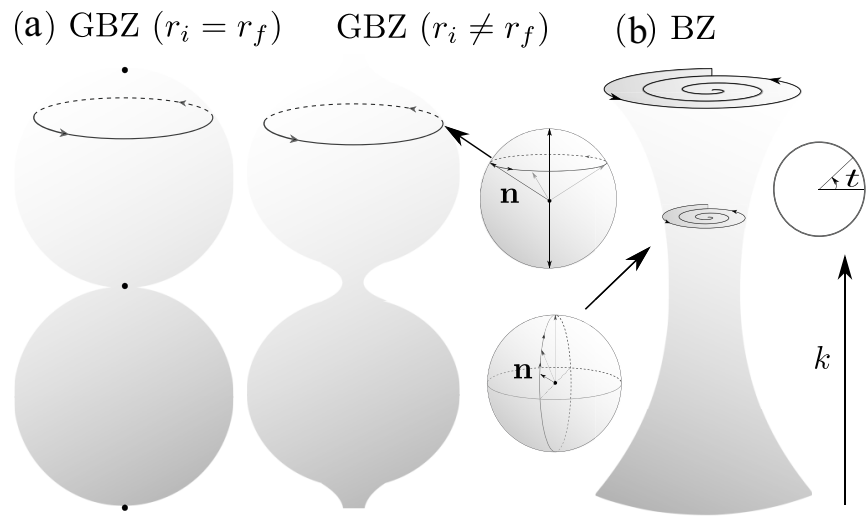

FIG. 1. Schematic illustration of (a) non-Bloch and (b) Bloch quench dynamics. (a) Non-Bloch quench dynamics in the GBZ are periodic in each $k$ sector, with either identical (left) or distinct (right) GBZs for the pre- and post-quench Hamiltonians [33,35]. The radius $r$ and generalized momentum $k$ of the GBZ, as well as the dynamic spin texture $\boldsymbol{n}(k, t)$, are defined in the main text. Black dots in the left panel indicate fixed points. (b) Bloch quench dynamics approach steady state in each $k$ sector. 
(a)

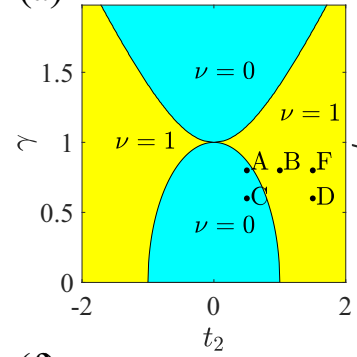

(f)

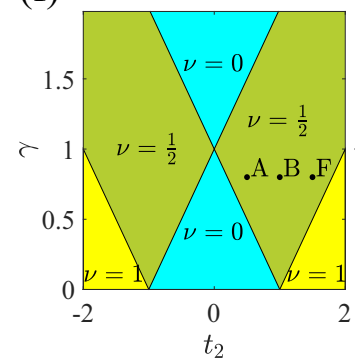

(b)

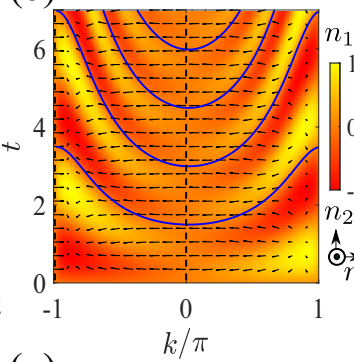

(g)

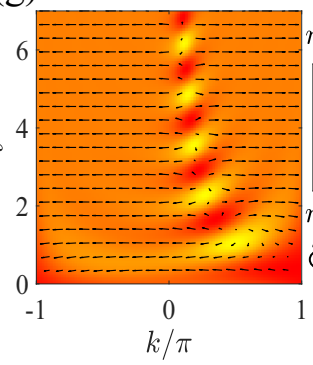

(c)

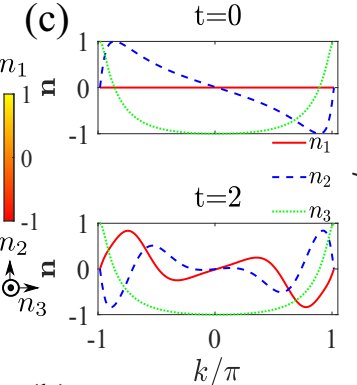

(h)

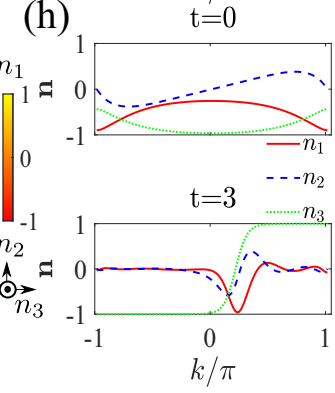

(d)

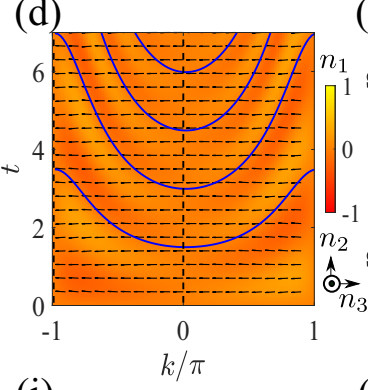

(i)

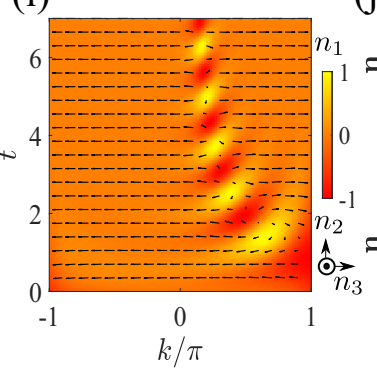

(e) $\quad t=0$

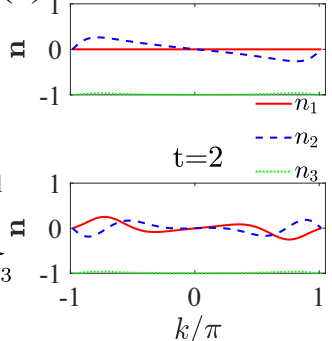

(j)

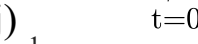

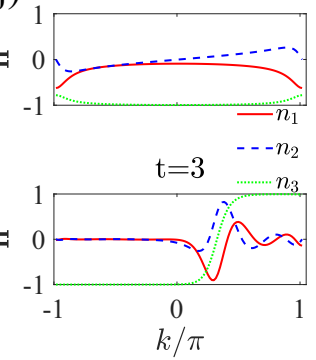

FIG. 2. Quench dynamics projected in the GBZ (top row) and BZ (lower row), where $t_{1}$ is taken as the unit of energy. (a) Non-Bloch topological phase diagram under OBC. Quenches are performed in the region with $\gamma<1$, where the eigenspectra are completely real. (b) $\boldsymbol{n}(k, t)$ in the generalized momentum-time space, for a quench from point A to F. Fixed points (dashed line) exist at $k_{m}=0$ with $c_{+}=0$, and $k_{m}= \pm \pi$ with $c_{-}=0$. (c) $\boldsymbol{n}(k, t)$ across the GBZ at different times $t$ for the quench in (b). (d) $\boldsymbol{n}(k, t)$ for a quench from point B to $\mathrm{F}$. Fixed points exist at $k_{m}=0, \pm \pi$ with $c_{+}=0$. (e) $\boldsymbol{n}(k, t)$ across the GBZ for the quench in (d). (f) Bloch topological phase diagram under PBC. Eigenspectra are complex throughout the phase diagram. (g) $\boldsymbol{n}(k, t)$ in the momentum-time space, for a quench from point A to F. (h) $\boldsymbol{n}(k, t)$ across the BZ for the quench in (g). (i) $\boldsymbol{n}(k, t)$ for a quench from point B to F. (j) $\boldsymbol{n}(k, t)$ across the BZ at different times $t$ for the quench in (i). Blue curves in (b) and (d) indicate integer multiples of $T_{k}$. The parameters for points A, B, and $\mathrm{F}$ are $t_{2}=0.5,1,1.5$, respectively, with $\gamma=0.8$. Points $\mathrm{C}$ and $\mathrm{D}$ are used in Fig. 3, with parameters $t_{2}=0.5,1.5$, respectively, and $\gamma=0.6$ for both. For the non-Bloch quench processes (top), $r_{i}=r_{f}=0.33$, whereas for the Bloch quenches (lower), $r_{i}=r_{f}=1$.

period $T_{k}=\pi / E_{k}^{f}$ [see Fig. 1(a)] [35,59]. Consequently, two different kinds of fixed points $k_{m}$ can appear in the GBZ, when the initial $\boldsymbol{n}\left(k_{m}, t=0\right)$ vector is aligned with either the north or south pole of the Bloch sphere, given respectively by $c_{ \pm}=\left\langle\chi_{k_{m}, \pm}^{f} \mid \psi_{k_{m},-}^{i}\right\rangle=0$.

These fixed points (if present), along with periodic dynamics, would divide the momentum-time space into multiple $S^{2}$ submanifolds. One can prove that a dynamic skyrmion structure can only emerge on a submanifold when it is pinned by fixed points of different kinds [59]. Further, when $H^{i}$ and $H^{f}$ possess distinct non-Bloch winding numbers but the same GBZ $\left(r_{i}=r_{f}\right)$, fixed points of different kinds necessarily emerge in pairs [59], which ultimately relates dynamic skyrmions in a quench with non-Bloch winding numbers of the static Hamiltonians. Similar results have been reported for quenches in the BZ, under either Hermitian $[33,34]$ or non-Hermitian, $\mathcal{P} T$-symmetric Hamiltonians [35,55] without skin effects. Albeit now, fixed points exist in the GBZ and the protecting symmetry for real eigenspectra is the non-Bloch $\mathcal{P} T$ symmetry. By contrast, if we project the quench dynamics here onto the BZ, by replacing $\left|\beta_{k, R / L}^{f}\right\rangle$ in $P_{k}$ with Bloch states, the dynamics would be approaching steady state, as Bloch spectra $E_{k, \pm}^{f}$ are necessarily complex for $k \in \mathrm{BZ}$ with $r^{f}=1$ [see Fig. 1(b)].

In Figs. 2(b) and 2(c), we show $\boldsymbol{n}(k, t)$, in the generalized momentum-time domain, for a quench between Hamiltonians with different non-Bloch winding numbers. As fixed points of different kinds exist at $k_{m}=0, \pm \pi$, arrays of momentum-time skyrmions appear, each characterized by a dynamic Chern number in the corresponding submanifold [33,34,59]. For a quench between Hamiltonians with the same non-Bloch winding numbers, fixed points still exist but are of the same kind, leading to the absence of skyrmions [see Figs. 2(d) and 2(e)]. For comparison, in Figs. 2(g)-2(j), we project the quench in the BZ, and the spin dynamics approach steady state, as expected. It is worth noting that, in our analysis, projected quench dynamics in different $k$ sectors are calculated independently. For a half-filled system initialized in the lower band, additional cross-coupling terms from other $k$ sectors would appear, but are vanishingly small in the thermodynamic limit when $r_{i}=r_{f}$ [59].

\section{BEYOND THE SIMPLE CASE}

For a quench between Hamiltonians with different GBZs, dynamics in different $k$ sectors of the GBZ are generically coupled, with nonvanishing cross-coupling contributions. Nevertheless, one can still perform the projection in Eq. (4), and focus solely on dynamics in each $k$ sector of $H^{f}$. Since fixed points no longer rigorously exist in this case [see Fig. 1(a)] [59], different submanifolds in the generalized momentum-time domain are no longer closed, and dynamic Chern numbers cannot be rigorously defined (except for the Chern number defined on the entire generalized momentumtime manifold which is always zero with unbroken $\mathcal{P} T$ 


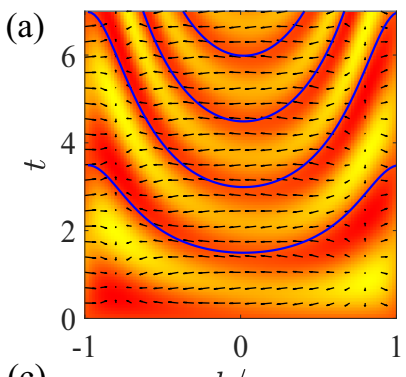

(c)

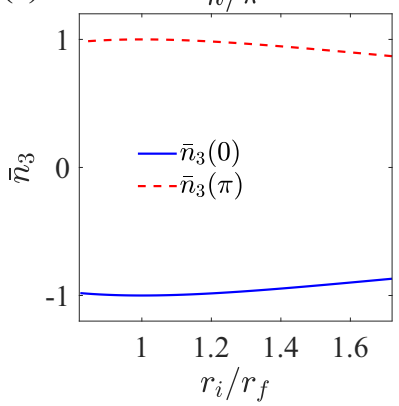

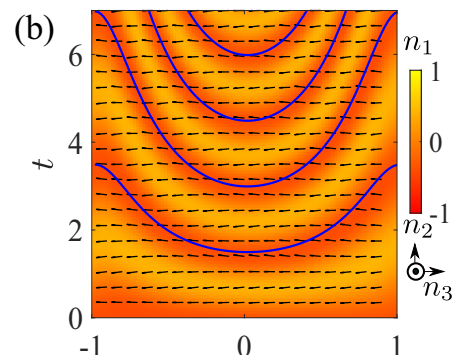

(d)

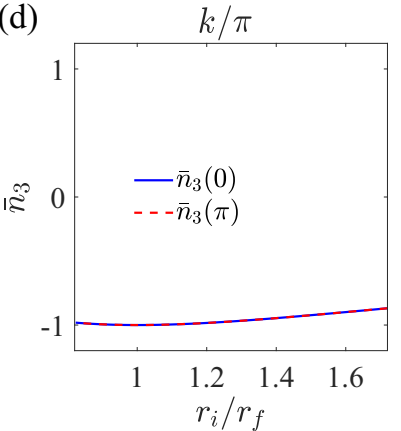

FIG. 3. Non-Bloch quench for $r_{i} \neq r_{f}$. (a) $\boldsymbol{n}(k, t)$ for a quench from point $\mathrm{C}$ in Fig. 2(a) $\left(r_{i}=0.5\right)$ to point $\mathrm{F}\left(r_{f}=0.33\right)$. (b) $\boldsymbol{n}(k, t)$ for a quench from point $\mathrm{D}$ in Fig. 2(a) $\left(r_{i}=0.5\right)$ to point F. (c) $\bar{n}_{3}(k=$ $0)$ and $\bar{n}_{3}(k=\pi)$ as functions of varying $r_{i} / r_{f}$, for non-Bloch quench processes ending at $\mathrm{F}$ and starting from points close to $\mathrm{C}$, with $v_{i}=$ 0 and a fixed $t_{2}=0.5$. (d) $\bar{n}_{3}(0)$ and $\bar{n}_{3}(\pi)$ with varying $r_{i} / r_{f}$, for quench processes ending at $\mathrm{F}$ and starting from points close to $\mathrm{D}$, with $v_{i}=1$ and a fixed $t_{2}=1.5$. We take $t_{1}=1$ as the unit of energy.

symmetry and hence uninformative). However, remnants of the dynamic skyrmions for $r_{i} / r_{f}=1$ (for $v_{i} \neq v_{f}$ ), or the lack thereof (for $v_{i}=v_{f}$ ), would carry over and leave identifiable signatures in the dynamic spin texture, over a considerable range of $r_{i} / r_{f}$.

We numerically confirm such a picture in Figs. 3(a) and $3(\mathrm{~b})$, where $r_{i} / r_{f}=1.5$. For both cases, the system is initialized in the lower band with occupied $\left|\psi_{k,-}^{i}\right\rangle$ of each $k$ sector, and under the same parameters with $v_{f}=1$. As expected, skyrmion-like structures only emerge in Fig. 3(a) where $v_{i} \neq v_{f}$. To capture key features of these structures away from $r_{i} / r_{f}=1$, we define the time-averaged spin component $\bar{n}_{3}(k)=\frac{1}{T_{k}} \int_{0}^{T_{k}} n_{3}(k, t) d t$, which should give $\bar{n}_{3}(0)=$ $-\bar{n}_{3}(\pi) \approx-1$ in the presence of skyrmion-like structures, and $\bar{n}_{3}(0)=\bar{n}_{3}(\pi) \approx-1$ otherwise. In Figs. 3(c) and 3(d), we show $\bar{n}_{3}(k)$ at $k=0$ and $k=\pi$ over a range of different $H^{i}$ (hence varying $r_{i} / r_{f}$ ), either with [see Fig. 3(c)] or without [see Fig. 3(d)] skyrmion-like structures. Crucially, knowing one of the non-Bloch winding numbers $v^{i / f}$, one can immediately infer the other with the knowledge of $\bar{n}_{3}\left(k_{m}\right)$. The quantity $\Delta \bar{n}_{3}=\bar{n}_{3}(0)-\bar{n}_{3}(\pi)$ thus serves as a feasible dynamic signal for the detection of non-Bloch topological invariants. Remarkably, similar conclusions can be drawn for quench dynamics between Hamiltonians with distinct, noncircular GBZs [59].

\section{NONUNITARY QUANTUM WALK}

Quantum-walk dynamics is an ideal platform for demonstrating non-Bloch quenches discussed here, particularly in
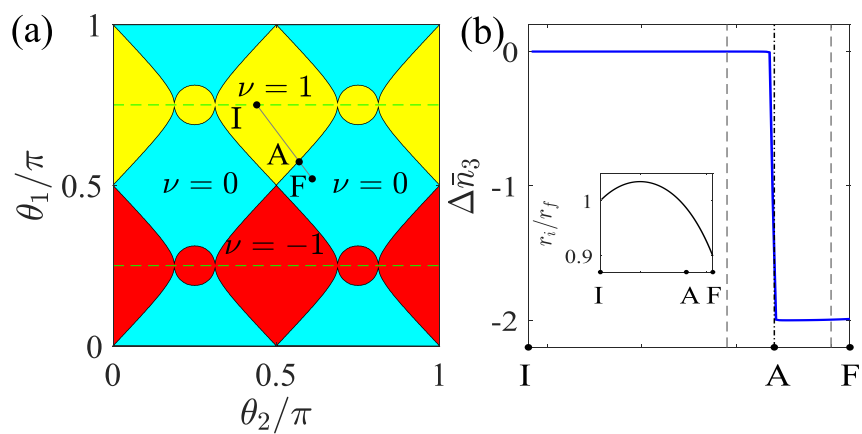

FIG. 4. (a) Non-Bloch topological phase diagram under OBC for the effective Hamiltonian $H$ of the Floquet operator $U$ in Eq. (6). The horizontal green dashed lines are locations where quasilocal eigenstates exist. (b) $\Delta \bar{n}_{3}$ evaluated for quench processes starting from $I$, and ending at different points along the line $\mathrm{I}-\mathrm{F}$ in (a). The vertical dashed and dash-dotted lines indicate, respectively, the Bloch and non-Bloch topological phase boundaries. Inset: Variation of $r_{i} / r_{f}$ as $H^{f}$ changes from $\mathrm{I}$ to $\mathrm{F}$. The parameters for points I, A, and $\mathrm{F}$ are $\left(\theta_{1}, \theta_{2}\right)=(0.75 \pi, 0.44 \pi),(0.573 \pi, 0.57 \pi),(0.52 \pi, 0.61 \pi)$, respectively.

light of the recent experimental observation of dynamic skyrmion structures therein [55]. For such a purpose, we consider a nonunitary quantum walk along a one-dimensional lattice, governed by the Floquet operator [28]

$$
U=R\left(\theta_{1}\right) S_{2} R\left(\theta_{2}\right) M R\left(\theta_{2}\right) S_{1} R\left(\theta_{1}\right),
$$

where $R\left(\theta_{i}\right)=\mathbf{1}_{w} \otimes e^{-i \theta_{i} \sigma_{y}}(i=1,2)$ acts on the internal coin states with basis $\{|A\rangle,|B\rangle\}$, and $M=\mathbf{1}_{w} \otimes\left(e^{\gamma}|A\rangle\langle A|+\right.$ $\left.e^{-\gamma}|B\rangle\langle B|\right)$. Here $\mathbf{1}_{w}=\sum_{x}|x\rangle\langle x|, x$ is the lattice-site index, and $\gamma$ is the gain-loss parameter. The shift operators $S_{1}=$ $\sum_{x}|x\rangle\langle x|\otimes| A\rangle\langle A|+| x+1\rangle\langle x|\otimes| B\rangle\langle B|$, and $S_{2}=\sum_{x} \mid x-$ $1\rangle\langle x|\otimes| A\rangle\langle A|+| x\rangle\langle x|\otimes| B\rangle\langle B|$.

For a discrete-time quantum walk, $U$ is repeatedly enforced upon an initial state. While state evolutions under $U$ are found to be localized near boundaries, due to the localization of all eigenstates under the non-Hermitian skin effect [28], the time evolution constitutes a stroboscopic simulation of the dynamics governed by an effective Hamiltonian $H$, with $U=$ $e^{-i H}$. Such a process can be further regarded as a quantum quench if the initial state is an eigenstate of a certain $H^{i}$, with $U^{i}=e^{-i H^{i}}$. In Fig. 4(a), we show the topological phase diagram of the effective Hamiltonian under OBC. Note that the GBZ here is always circular, which simplifies the problem.

To facilitate experimental implementation, we choose a quasilocal initial state $\left|\Psi^{i}\right\rangle \propto|0\rangle \otimes(|A\rangle+|B\rangle)+2 i / r_{i} \mid-$ $1\rangle \otimes|A\rangle+1 / r_{i}^{2}|-2\rangle \otimes(-|A\rangle+|B\rangle)$, which is an eigenstate of $U^{i}$ at point $I$ in Fig. 4(a). Writing the initial state in the GBZ as $\left|\Psi^{i}\right\rangle=\sum_{k}\left|\beta_{k, R}^{i}\right\rangle \otimes\left|\psi_{k}^{i}\right\rangle$ and evolving it repeatedly with $U^{f}$, we follow the prior practice of projecting the timedependent density matrix into different $k$ sectors of the GBZ of $H^{f}$ (with $U^{f}=e^{-i H^{f}}$ ), from which $\boldsymbol{n}(k, t)$ can be extracted [59]. We then vary parameters of $H^{f}$ along the line I-F in Fig. 4(a), and show the change of $\Delta \bar{n}_{3}$ in Fig. 4(b). Despite the deviation of $r_{i} / r_{f}$ from unity [inset of Fig. 4(b)], $\Delta \bar{n}_{3}$ features an abrupt jump at the non-Bloch topological phase 
transition, thus providing a sensitive signal for the detection of non-Bloch winding number $v_{f}$, once $v_{i}$ is known.

\section{CONCLUSION}

We show that projecting quench dynamics onto the GBZ reveals dynamic topological structures that can be used as signals for detecting non-Bloch topological invariants. In the spirit of the non-Bloch quench dynamic discussed here, it is hopeful that various previously observed dynamic topological structures in unitary quench processes, such as vortices [50], links [51], and rings [52], can be explored in systems with non-Hermitian skin effects, which would then serve to detect non-Bloch topological invariants for more general models in higher dimensions.

\section{ACKNOWLEDGMENTS}

We acknowledge helpful discussions with Tian-Shu Deng. This work is supported by the National Natural Science Foundation of China (Grants No. 11974331, No. 11674306, and No. 92065113) and the National Key R\&D Program (Grants No. 2016YFA0301700 and No. 2017YFA0304100).

\section{APPENDICES}

Here we provide more details on the non-Hermitian SuSchieffer-Heeger (SSH) model, the projection of quench dynamics in the generalized Brillouin zone (GBZ), derivation for the conditional existence of fixed points and dynamic Chern numbers, analysis and numerical calculations demonstrating the persistence of skyrmions-like structures in more general settings, as well as theoretical and numerical analysis of quantum-walk dynamics.

\section{APPENDIX A: NON-HERMITIAN SSH MODEL WITH SKIN EFFECTS}

For the non-Hermitian SSH model [Eq. (1) in the main text] under the open boundary condition (OBC), all of its nominal bulk eigenstates are localized near the boundaries (for $\gamma \neq 0$ ) [11], and Bloch quasimomenta of the Brillouin zone (BZ) are no longer good bulk quantum numbers even in the thermodynamic limit. Central to our analysis here is the projection of dynamics onto GBZs characterized by the generalized momentum $k$ associated with the biorthogonal spatial basis,

$$
\left|\beta_{k, R(L)}\right\rangle=\frac{1}{\sqrt{N}} \sum_{n} r^{ \pm n} e^{i k n}|n\rangle,
$$

where $r$ is the radius the GBZ, $k$ is identified as the generalized momentum of the GBZ, and the basis states satisfy $\left\langle\beta_{k, L} \mid \beta_{k^{\prime}, R}\right\rangle=\delta_{k, k^{\prime}}$ and $\sum_{k}\left|\beta_{k, R}\right\rangle\left\langle\beta_{k, L}\right|=1$. While any singleparticle state of the system can be generically written as $|\Psi\rangle=$ $\sum_{k}\left|\beta_{k, R}\right\rangle \otimes\left|\psi_{k}\right\rangle$, with $\left|\psi_{k}\right\rangle=\left\langle\beta_{k, L} \mid \Psi\right\rangle$ its internal state in the sublattice space $\{|A\rangle,|B\rangle\}$, we introduce the projection operators $P_{k}=\left|\beta_{k, R}\right\rangle\left\langle\beta_{k, L}\right| \otimes \mathbf{1}_{\alpha}$ and $Q_{k}=\mathbf{1}-P_{k}$, where $\mathbf{1}_{\alpha}$ and $\mathbf{1}$ are the identity operators in the sublattice space and the full Hilbert space of the system, respectively. This enables us to write, in the thermodynamic limit, $H=P_{k} H P_{k}+Q_{k} H Q_{k}=$
$H_{k}+Q_{k} H Q_{k}$, where $H$ is given in Eq. (1) of the main text, $H_{k}=P_{k} H P_{k}$, and we have used the fact that $P_{k} H Q_{k}$ and $Q_{k} H P_{k}$ vanish in the thermodynamic limit, though neither of them are exactly zero for a finite-size system.

Under the sublattice basis defined above, $H_{k}$ can be written in the matrix form

$$
H_{k}=\left(\begin{array}{cc}
0 & t_{1}+\gamma+t_{2} r^{-1} e^{-i k} \\
t_{1}-\gamma+t_{2} r e^{i k} & 0
\end{array}\right) .
$$

The right and left eigenstates of $H_{k}$ are defined through $H_{k}\left|\psi_{k, \pm}\right\rangle= \pm E_{k}\left|\psi_{k, \pm}\right\rangle$ and $H_{k}^{\dagger}\left|\chi_{k, \pm}\right\rangle= \pm E_{k}^{*}\left|\chi_{k, \pm}\right\rangle$, respectively. They further satisfy the biorthogonal and completeness relations: $\left\langle\chi_{k, \mu} \mid \psi_{k, \nu}\right\rangle=\delta_{\mu \nu}$ and $\sum_{\mu}\left|\psi_{k, \mu}\right\rangle\left\langle\chi_{k, \mu}\right|=\mathbf{1}_{\alpha}$. Throughout the work, we mainly focus on the regime with $t_{1}>\gamma$, where, under an exact non-Bloch parity-time $(\mathcal{P} T)$ symmetry, the eigenspectrum $E_{k}$ is completely real. Here $E_{k}=\sqrt{\bar{t}_{1}^{2}+t_{2}^{2}+2 \bar{t}_{1} t_{2} \cos k}$, with $\bar{t}_{1}=\sqrt{\left(t_{1}-\gamma\right)\left(t_{1}+\gamma\right)}$, and \pm are the band indices. The eigenstates are given as

$$
\begin{aligned}
& \left|\psi_{k, \pm}\right\rangle=\left(\begin{array}{c} 
\pm 1 / \sqrt{\bar{t}_{1}^{2}+t_{2}^{2}+2 \bar{t}_{1} t_{2} \cos k} \\
r /\left(\bar{t}_{1}+t_{2} e^{-i k}\right)
\end{array}\right) \\
& \left|\chi_{k, \pm}\right\rangle=\left(\begin{array}{c} 
\pm 1 / \sqrt{\bar{t}_{1}^{2}+t_{2}^{2}+2 \bar{t}_{1} t_{2} \cos k} \\
1 / r\left(\bar{t}_{1}+t_{2} e^{-i k}\right)
\end{array}\right) .
\end{aligned}
$$

While the biorthogonal spatial basis of the GBZ can be seen as the non-Bloch analog of Bloch waves, the complication of non-Bloch quench dynamics, i.e., the analysis of quench dynamics in the GBZ, comes from the general nonorthogonality of spatial basis states of the pre- and postquench Hamiltonians, even if they are of the same $k$ sector.

\section{APPENDIX B: QUENCH DYNAMICS AND FIXED POINTS IN THE GBZ}

Consider a quench process initialized in the lower band of the initial Hamiltonian $H^{i}$, i.e., with all eigenstates $\left|\psi_{k,-}^{i}\right\rangle$ occupied. The initial state in a given $k$ sector is then $\left|\Psi_{k}^{i}\right\rangle=$ $\left|\beta_{k, R}^{i}\right\rangle \otimes\left|\psi_{k,-}^{i}\right\rangle$, where $\left|\beta_{k, R}^{i}\right\rangle$ is the right spatial basis characterized, according to Eq. (A1), by the GBZ radius $r_{i}$ of $H^{i}$. Evolving the initial state with the Hamiltonian $H^{f}$, the time-dependent density matrix is given by

$$
\rho(t)=e^{-i H^{f} t}\left|\Psi_{k}^{i}\right\rangle\left\langle\Psi_{k}^{i}\right| e^{i H^{f^{\dagger}} t} .
$$

Importantly, when $\gamma \neq 0,\left\langle\beta_{k, L}^{f} \mid \beta_{k^{\prime}, R}^{i}\right\rangle \neq 0$ (and $\left\langle\beta_{k, R}^{f} \mid \beta_{k^{\prime}, L}^{i}\right\rangle \neq$ $0)$, and dynamics in different $k$ sectors are coupled. Here $\left|\beta_{k, R / L}^{f}\right\rangle$ are the right or left spatial bases associated with the GBZ of $H^{f}$ according to Eq. (A1).

To overcome this difficulty, we project the dynamics onto the GBZ of $H^{f}$, such that the density matrix in each $k$ sector becomes

$$
\rho(k, t)=P_{k} \rho(t) P_{k}^{\dagger}=\mathcal{N}^{2} e^{-i H_{k}^{f} t}\left|\psi_{k,-}^{i}\right\rangle\left\langle\psi_{k,-}^{i}\right| e^{i H_{k}^{f \dagger} t},
$$

where $\quad P_{k}=\left|\beta_{k, R}^{f}\right\rangle\left\langle\beta_{k, L}^{f}\right| \otimes \mathbf{1}_{\alpha}, \quad Q_{k}=\mathbf{1}-P_{k}, \quad \mathcal{N}=$ $\left\langle\beta_{k, L}^{f} \mid \beta_{k, R}^{i}\right\rangle=\frac{1}{N} \sum_{n}\left(\frac{r_{i}}{r_{f}}\right)^{n}$, and we have used $H_{k}^{f}=P_{k} H^{f} P_{k}$, $P_{k} H^{f} Q_{k}=0$. 
As discussed in the main text, the projected dynamics can be visualized on a Bloch sphere through

$$
\boldsymbol{n}(k, t)=\frac{\operatorname{Tr}[\rho(k, t) \eta \boldsymbol{\tau}]}{\operatorname{Tr}[\rho(k, t) \eta]},
$$

where $\eta=\sum_{\mu}\left|\chi_{k, \mu}^{f}\right\rangle\left\langle\chi_{k, \mu}^{f}\right|$ is the metric operator, $\boldsymbol{\tau}=$ $\left(\tau_{1}, \tau_{2}, \tau_{3}\right)$, with $\tau_{i}=\sum_{\mu \nu= \pm}\left|\psi_{k, \mu}^{f}\right\rangle \sigma_{i}^{\mu \nu}\left\langle\chi_{k, \nu}^{f}\right|(i=0,1,2,3)$. These definitions ensure that $\boldsymbol{n}(k, t)=\left(n_{1}, n_{2}, n_{3}\right)$ is always a real, unit vector.

When the eigenspectrum $E_{k}^{f}$ of $H^{f}$ is real, we have $[35,55]$

$$
\begin{gathered}
n_{1}=\left(c_{+}^{*} c_{-} e^{2 i E_{k}^{f} t}+\text { c.c. }\right) / n_{0}, \\
n_{2}=i\left(c_{+}^{*} c_{-} e^{2 i E_{k}^{f} t}-\text { c.c. }\right) / n_{0}, \\
n_{3}=\left(c_{+}^{*} c_{+}-c_{-}^{*} c_{-}\right) / n_{0},
\end{gathered}
$$

where $c_{\mu}(k)=\left\langle\chi_{k, \mu}^{f} \mid \psi_{k,-}^{i}\right\rangle$, and $n_{0}=c_{+}^{*} c_{+}+c_{-}^{*} c_{-}$. In this case, $n_{3}$ is time independent, and $\boldsymbol{n}(k, t)$ in a given $k$ sector rotates around the north pole of the Bloch sphere with a period $T_{k}=\pi / E_{k}^{f}$ [see Fig. 1(a) of the main text], where the north and south poles are given by the fixed-point conditions $c_{-}\left(k_{m}\right)=0$ and $c_{+}\left(k_{m}\right)=0$, respectively. Here $k_{m}$ is the location of the fixed point, where the local density matrix does not evolve in time.

When $E_{k}^{f}$ is purely imaginary,

$$
\begin{gathered}
n_{1}=\left(c_{+}^{*} c_{-}+\text {c.c. }\right) / n_{0}, \\
n_{2}=i\left(c_{+}^{*} c_{-}-\text {c.c. }\right) / n_{0}, \\
n_{3}=\left(c_{+}^{*} c_{+} e^{-i 2 E_{k}^{f} t}-c_{-}^{*} c_{-} e^{i 2 E_{k}^{f} t}\right) / n_{0},
\end{gathered}
$$

where $n_{0}=c_{+}^{*} c_{+} e^{-i 2 E_{k}^{f} t}+c_{-}^{*} c_{-} e^{i 2 E_{k}^{f} t}$. As $t$ tends to infinity, $\boldsymbol{n}(k, t)$ asymptotically approaches either the north (for $\operatorname{Im} E_{k}>0$ ) or south (for $\operatorname{Im} E_{k}<0$ ) pole [see Fig. 1(b) of the main text].

Further, from the analytical expression of $c_{\mu}$,

$$
\begin{aligned}
c_{ \pm}(k)= & \mp \frac{1}{\sqrt{\bar{t}_{1 i}^{2}+t_{2 i}^{2}+2 \bar{t}_{1 i} t_{2 i} \cos k}} \frac{1}{\sqrt{\bar{t}_{1 f}^{2}+t_{2 f}^{2}+2 \bar{t}_{1 f} t_{2 f} \cos k}} \\
& +\frac{r_{i}}{r_{f}} \frac{1}{\left(\bar{t}_{1 i}+t_{2 i} e^{-i k}\right)\left(\bar{t}_{1 f}+t_{2 f} e^{i k}\right)},
\end{aligned}
$$

we see that when $r_{i}=r_{f}$, there are always two fixed points at $k_{m}=0$ and $k_{m}=\pi$, respectively.

The existence of fixed points and periodic dynamics in the $k$ sectors (under a completely real $E_{k}^{f}$ ) divide the generalized momentum-time space into a series of $S^{2}$ submanifolds, on each of which a quantized, dynamic Chern number can be defined,

$$
C_{m n}=\frac{1}{4 \pi} \int_{k_{m}}^{k_{n}} d k \int_{0}^{T_{k}} d t\left[\boldsymbol{n}(k, t) \times \partial_{t} \boldsymbol{n}(k, t)\right] \cdot \partial_{k} \boldsymbol{n}(k, t),
$$

where $k_{m}, k_{n} \in\{0, \pm \pi\}$ are two adjacent fixed points. It is straightforward to show that when $c_{+}\left(k_{m}\right)=0$ and $c_{-}\left(k_{n}\right)=$ $0, C_{m n}=1$; when $c_{-}\left(k_{m}\right)=0$ and $c_{+}\left(k_{n}\right)=0, C_{m n}=-1$; otherwise, $C_{m n}=0$. Hence, if we categorize fixed points into two different kinds, with either $c_{+}\left(k_{m}\right)=0$ or $c_{-}\left(k_{m}\right)=0$, the dynamic Chern number can only take nonzero values when the corresponding submanifold is pinned by fixed points of different kinds. While these dynamic Chern numbers serve as skyrmion numbers for the dynamic skyrmions in the generalized momentum-time space shown in the main text, they are linked with the non-Bloch winding numbers of the pre- and post-quench Hamiltonians. Specifically, from Eq. (B10) and in combination with the expression for non-Bloch winding numbers [Eq. (2) of the main text], we find that, for $v_{i} \neq v_{f}$, two fixed points of different kinds emerge at $0, \pi$, ensuring the appearance of dynamic skyrmion structure. However, for $v_{i}=v_{f}$, the two fixed points at 0 and $\pi$ are of the same kind, and dynamic skyrmions are absent. We note that Eq. (B10) as well as the conclusions above are conditional on the reality of the eigenspectra $E_{k}^{i, f}$ of $H_{k}^{i, f}$, which is the regime of study here.

For the more general case of $r_{i} \neq r_{f}$, i.e., the GBZs of the pre- and post-quench Hamiltonians do not match, fixed points do not exist in general. First, this can be concluded by observing that $c_{ \pm}(k) \neq 0$ in Eq. (B10). Furthermore, as a state initialized in a given $k$ sector would proliferate into other $k$ sectors during the time evolution, Eq. (B10) cannot be used to characterize dynamics for the quench dynamics of a topological system at half filling, where all $k$ sectors of the lower band are occupied initially at $t=0$. However, as long as $r_{i} / r_{f}$ does not significantly deviate from unity, the impact of the these cross-coupling terms is typically small [see, for instance, a related analysis following Eq. (D1)]; and in each $k$ sector, $\boldsymbol{n}(0, t)$ and $\boldsymbol{n}(\pi, t)$ (for completely real $E_{k}^{f}$ ) precess closely around the poles of the Bloch sphere, and fixed points exist in a perturbative sense. Although dynamic Chern numbers cannot be rigorously defined in this case, global signatures of dynamic skyrmions (for $v_{i} \neq v_{f}$ ), or the lack thereof (for $v_{i}=v_{f}$ ), would still be discernible beyond $r_{i}=r_{f}$. In fact, in the following section, we show that dynamic skyrmions even persist when GBZs of neither Hamiltonian are circular. Such an understanding underlies our proposal for a general detection scheme of non-Bloch topological invariants.

\section{APPENDIX C: NON-HERMITIAN SSH MODEL WITH NEXT-NEAREST-NEIGHBOR HOPPING}

To further illustrate the applicability of our scheme on models with more general GBZs, we consider the quench dynamics of a non-Hermitian SSH model with next-nearestneighbor hopping, i.e., with an additional term

$$
H_{n n}=\sum_{n}\left(t_{3}|n, A\rangle\langle n+1, B|+\text { H.c. }\right),
$$

where $t_{3}$ is the hopping rate. For a non-Hermitian SSH model with a finite $t_{3}$, the GBZ is no longer a circle on the complex plane. The spatial basis involves a $k$-dependent radius

$$
\left|\beta_{k, R(L)}\right\rangle=\frac{1}{\sqrt{N}} \sum_{n} r^{ \pm n}(k) e^{i k n}|n\rangle, \quad k \in(-\pi, \pi],
$$

which does not satisfy the biorthogonal relations in the main text, i.e., $\left\langle\beta_{k^{\prime}, L} \mid \beta_{k, R}\right\rangle \neq 0$. 
(a)

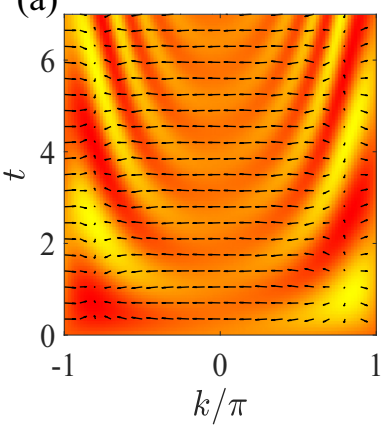

(b)

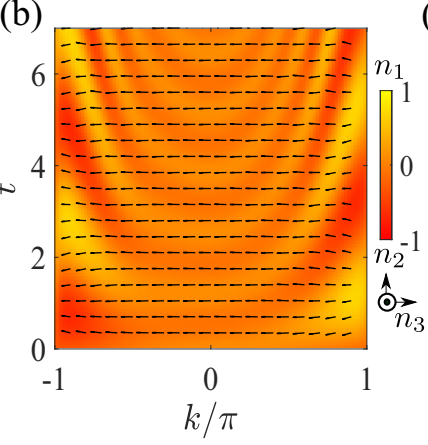

(c)

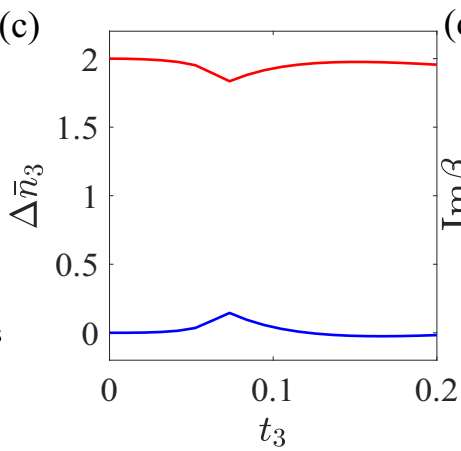

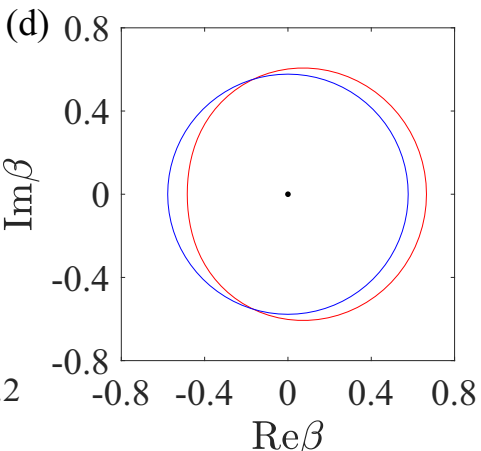

FIG. 5. (a), (b): Spin texture $\boldsymbol{n}(k, t)$ on the generalized momentum-time space for non-Bloch quench dynamics under non-Hermitian SSH model with next-nearest-neighbor hopping, with $\gamma=0.5$. (a) Quench from $H^{i}$ with $t_{2}^{i}=0.5$ and $t_{3}^{i}=0$ (non-Bloch winding number $v^{i}=0$ ) to $H^{f}$ with $t_{2}^{f}=1.5$ and $t_{3}^{f}=0.05$ (non-Bloch winding number $v^{f}=1$ ). (b) Quench from $t_{2}^{i}=1$ and $t_{3}^{i}=0$ (non-Bloch winding number $v^{i}=1$ ) to $t_{2}^{f}=1.5$ and $t_{3}^{f}=0.05$. (c) $\Delta \bar{n}_{3}$ with increasing $t_{3}^{f}$ and a fixed $t_{2}^{f}=1.5$. The initial parameters are $t_{2}^{i}=0.5$ (red) and $t_{2}^{i}=1$ (blue), respectively. (d) GBZs of $H^{f}$ in (c), with $t_{3}^{f}=0$ (blue) and $t_{3}^{f}=0.05$ (red), respectively. GBZs of $H^{i}$ for both cases coincide with the blue circle. We take $t_{1}=1$ as the unit of energy.

However, when $t_{3}$ is small, the GBZ does not deviate too much from a circle, remnants of the dynamic skymion structures still exist for $v_{i} \neq v_{f}$, and they can still be used to determine the post-quench non-Bloch

$$
H_{k}=\left(\begin{array}{c}
0 \\
t_{1}-\gamma+t_{2} r e^{i k}+t_{3} r^{-1} e^{-i k}
\end{array}\right.
$$

and one can perform the same analysis outlined above to study projected quench dynamics in the GBZ of $H^{f}$.

In Fig. 5, we show the numerical simulation of such a quench process, with the state in each $k$ sector of the GBZ initialized in the lower band of $H_{k}^{i}$, and projecting the dynamics onto the GBZ of $H^{f}$. In Fig. 5(a), $v_{i}=0$ and $v_{f}=1$, so that skyrmion-like structures are visible. By contrast, in Fig. 5(b), $v_{i}=1$ and $v_{f}=1$, and no visible skyrmion structures are present. Furthermore, we characterize in Fig. 5(c) the variation of GBZs with increasing $t_{3}$, and show the resulting $\bar{n}_{3}$ as a function of $t_{3}$ in Fig. 5(d). Similarly to the main text, $\bar{n}_{3}=$ $\left(1 / T_{k}^{\prime}\right) \int_{0}^{T_{k}^{\prime}}\left[n_{3}(0, t)-n_{3}(\pi, t)\right] d t$, where $T_{k}^{\prime}=\operatorname{Re} E_{k}^{f}$, is used as the signature for the skyrmion-like structure. Apparently, $\bar{n}_{3} \sim 2\left(\bar{n}_{3} \sim 0\right)$ for $v_{i} \neq v_{f}\left(v_{i}=v_{f}\right)$, for over a considerable range of $t_{3}$.

\section{APPENDIX D: QUENCH DYNAMICS IN A QUANTUM WALK}

As discussed in the main text, we consider quantum walk dynamics governed by the Floquet operator $U$ given by Eq. (6). Under an OBC, the GBZ of $U$ is a circle on the complex plane, its radius $r=\sqrt{\left|\frac{\cosh \gamma \cos 2 \theta_{2}-\sinh \gamma}{\cosh \gamma \cos 2 \theta_{2}+\sinh \gamma}\right|}$ [32].

We start from a localized initial state $\left|\Psi^{i}\right\rangle \propto|0, A\rangle+$ $|0, B\rangle+2 i / r_{i}|-1, A\rangle+1 / r_{i}^{2}(-|-2, A\rangle+|-2, B\rangle)$, which is an eigenstate of an initial Floquet operator $U^{i}$ with $\theta_{1}^{i}=$ $3 \pi / 4$ and $\theta_{2}^{i}=0.44 \pi$. Formally writing the initial state as $\left|\Psi_{i}\right\rangle=1 / \sqrt{N} \sum_{k}\left|\Psi_{k}^{i}\right\rangle$, where $\left|\Psi_{k}^{i}\right\rangle=\left|\beta_{k, R}^{i}\right\rangle \otimes\left|\psi_{k}^{i}\right\rangle$, we winding number once that of the initial Hamiltonian is known.

More concretely, the projected Hamiltonian in a given $k$ sector is given by

$$
\left.\begin{array}{c}
t_{1}+\gamma+t_{2} r^{-1} e^{-i k}+t_{3} r e^{i k} \\
0
\end{array}\right)
$$

evolve it under the Floquet operator $U=e^{-i H^{f} t}$. The timeevolved state is then $|\Psi(t)\rangle=\sum_{k} e^{-i H^{f} t}\left|\Psi_{k}^{i}\right\rangle$. Projecting the dynamics onto the GBZ of the final effective Hamiltonian $H^{f}$, we have

$$
\begin{aligned}
|\psi(k, t)\rangle & =P_{k}|\Psi(t)\rangle \\
& =P_{k} \sum_{k} e^{-i H^{f} t}\left|\Psi_{k}^{i}\right\rangle \\
& =\mathcal{N}(0)\left(e^{-i H_{k}^{f} t}\left|\psi_{k}^{i}\right\rangle+\sum_{k^{\prime} \neq k} \frac{\mathcal{N}\left(k-k^{\prime}\right)}{\mathcal{N}(0)} e^{-i H_{k}^{f} t}\left|\psi_{k^{\prime}}^{i}\right\rangle\right),
\end{aligned}
$$

where $\mathcal{N}\left(k-k^{\prime}\right)=\left\langle\beta_{k, L}^{f} \mid \beta_{k^{\prime}, R}^{i}\right\rangle=\frac{1}{N} \sum_{n}\left(\frac{r_{i}}{r_{f}}\right)^{n} e^{-i\left(k-k^{\prime}\right) n}$. Since $\left|\mathcal{N}\left(k-k^{\prime}\right) / \mathcal{N}(0)\right| \sim\left|\left(r_{i}-r_{f}\right) /\left(r_{i}+r_{f}\right)\right|$, the second term on the right-hand side of Eq. (D1) vanishes for $r_{i}=r_{f}$, and is negligibly small for $\left|r_{i}-r_{f}\right| \ll r_{i}+r_{f}$. We note that a similar situation should occur for the quench dynamics of lattice models at half filling, as we discussed previously.

To see the relation between non-Bloch winding numbers of the effective Hamiltonian and non-Bloch quench dynamics, we first project the Floquet operator $U$ onto the GBZ,

$$
U(k)=P_{k} U P_{k},
$$

which, under the parameters considered in the main text $\left|\cos 2 \theta_{2}\right|>|\tanh \gamma|$, can be decomposed as

$$
U(k)=d_{0} \sigma_{0}-i d_{1} \sigma_{1}-i d_{2} \sigma_{2}-i d_{3} \sigma_{3},
$$




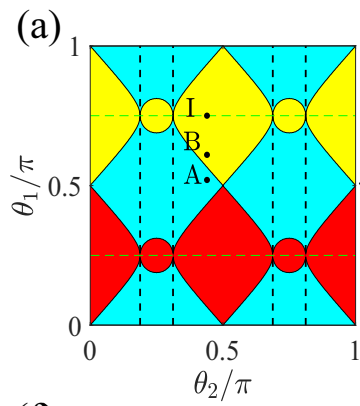

(f)

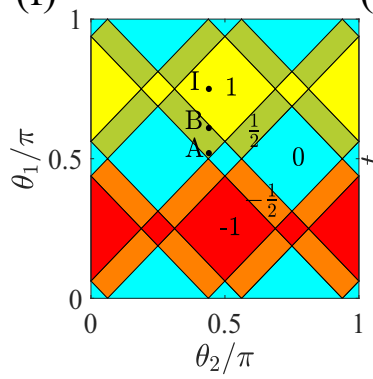

(b)

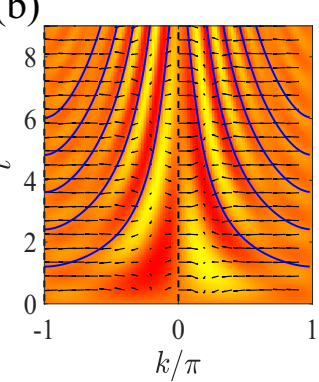

(g)

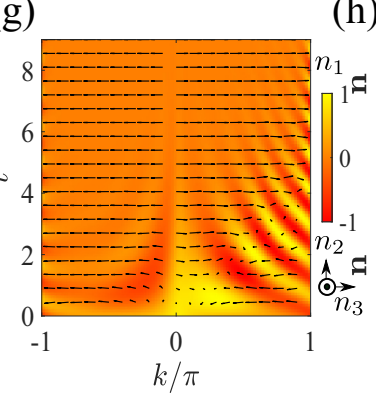

(c)

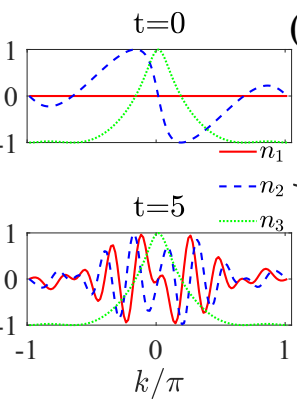

(h)

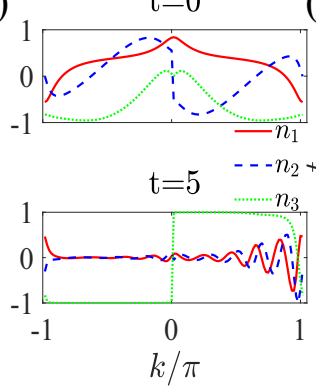

(d)

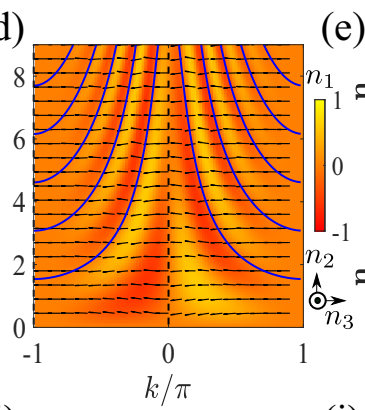

(i)

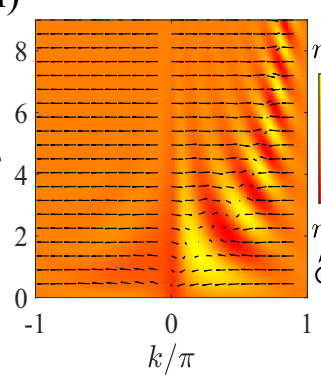

(e)

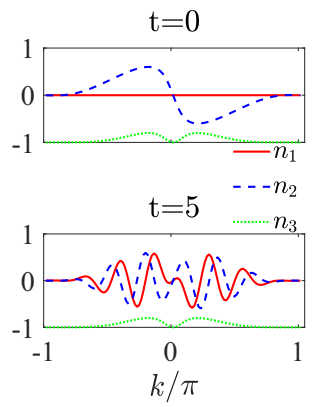

(j)

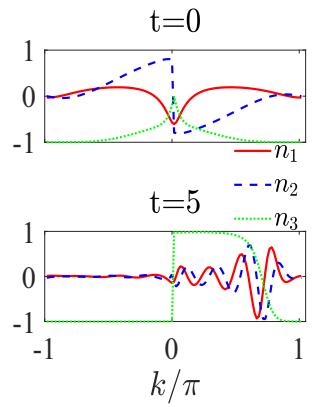

FIG. 6. Stroboscopic simulation of quench dynamics using a quantum walk governed by $U$ in Eq. (6), with $\gamma=0.4$. Quench dynamics are projected in (top) the GBZ and (lower) BZ, respectively. (a) Non-Bloch topological phase diagram of the effective Hamiltonian $H$ under OBC, where $U=e^{-i H}$. The horizontal green lines represent locations of localized eigenstates. The vertical dashed lines are the non-Bloch exceptional lines separating the exact non-Bloch $\mathcal{P} T$-symmetric and $\mathcal{P} T$-broken regimes, where we only focus on the $\mathcal{P} T$-unbroken regime. Regions filled in yellow, cyan, and red respectively feature non-Bloch winding numbers $v=1,0,-1$. (b) Dynamic spin texture $\boldsymbol{n}(k, t)$ in the generalized momentum-time space, for a quantum quench from point I (with non-Bloch winding number $v_{i}=1$ ) to A (with non-Bloch winding number $v_{f}=0$ ). (c) $\boldsymbol{n}(k, t)$ in the GBZ at different times $t$ for the quench in (b). (d) Spin texture $\boldsymbol{n}(k, t)$ for a quench from point I to B (with non-Bloch winding number $v_{f}=1$ ). No skyrmion structures are present, in contrast to (b). (e) Components of $\boldsymbol{n}(k, t)$ in the GBZ at different times $t$ for the quench in (d). (f) Bloch topological phase diagram under PBC. Eigenspectra are complex throughout the phase diagram. Bloch winding numbers are marked in the phase diagram, where regions with different winding numbers are colored differently. ( $\mathrm{g}$ ) Dynamic spin texture $\boldsymbol{n}(k, t)$ in the momentum-time space (BZ), for a quantum quench from point I (with Bloch winding number $v_{i}=1$ ) to A (with Bloch winding number $v_{f}=1 / 2$ ). (h) Components of $\boldsymbol{n}(k, t)$ in the BZ at different times $t$ for the quench in (g). (i) Spin texture $\boldsymbol{n}(k, t)$ in the momentum-time space for a quench from point I to B (with Bloch winding number $\left.v_{f}=1 / 2\right)$. (j) Components of $\boldsymbol{n}(k, t)$ in the BZ at different times $t$ for the quench in (i). The parameters for points A, B, and I are $\left(\theta_{1}, \theta_{2}\right)=(0.52 \pi, 0.44 \pi),(0.61 \pi, 0.44 \pi),(0.75 \pi, 0.44 \pi)$, respectively.

where

$$
\begin{gathered}
d_{0}=\lambda \cos 2 \theta_{1} \cos k-\sin 2 \theta_{1} \sin 2 \theta_{2} \cosh \gamma, \\
d_{1}=0, \\
d_{2}=\lambda \sin 2 \theta_{1} \cos k+\cos 2 \theta_{1} \sin 2 \theta_{2} \cosh \gamma, \\
d_{3}=-\lambda \sin k .
\end{gathered}
$$

Here $\quad \lambda=\left[\left(\cosh \gamma \cos 2 \theta_{2}-\sinh \gamma\right)\left(\cosh \gamma \cos 2 \theta_{2}+\right.\right.$ $\sinh \gamma)]^{1 / 2}$ when $\cos 2 \theta_{2}>|\tanh \gamma| ;$ and $\lambda=$ $-\left[\left(\cosh \gamma \cos 2 \theta_{2}-\sinh \gamma\right)\left(\cosh \gamma \cos 2 \theta_{2}+\sinh \gamma\right)\right]^{1 / 2}$ when $\cos 2 \theta_{2}<-|\tanh \gamma|$.

The non-Bloch winding number of the effective Hamiltonian $H$, with $U=e^{-i H}$, is calculated through [28]

$$
v=\frac{1}{2 \pi} \int d k \frac{-d_{3} \partial_{k} d_{2}+d_{2} \partial_{k} d_{3}}{d_{2}^{2}+d_{3}^{2}},
$$

which reduces to the Bloch winding number for $\gamma=0$.

Notably, $U(k)$ is unitary and chiral symmetric with $\sigma_{x} U(k) \sigma_{x}=U^{-1}(k)$, where $\sigma_{x}$ is the chiral symmetry operator. It follows that $H_{k}^{f}$ in Eq. (D1) is Hermitian and chiral symmetric, and the proof regarding the existence of topolog- ical fixed points and dynamic Chern number (hence dynamic skyrmions) is essentially the same as that in Ref. [33]. Importantly, these conclusions are exact for $r_{i}=r_{f}$, when the second term in Eq. (D1) is zero, and approximately true when the term is negligibly small.

In Fig. 6, we perform a numerical simulation of quantum walk dynamics, starting from a localized state in the bulk. The quench dynamics are projected onto the GBZ in the first row of Fig. 6, and onto the BZ in the second row. We focus here on the case with $r_{i}=r_{f}$, as more general cases are already shown in the main text. Here dynamic skyrmions emerge in Fig. 6(b), where $v_{i}=1$ and $v_{f}=0$. In Fig. 6(d), on the other hand, no dynamic skyrmions are present, as $v_{i}=1$ and $v_{f}=1$. Therefore, if either the non-Bloch winding number of the initial or the final effective Hamiltonian is known, one can infer the value of the other one by the observation of spin textures. Consistently with these conclusions, we find that fixed points exist at $k_{m}=0$ and $k_{m}=\pi$ for both cases [see Figs. 6(c) and 6(e)], but they are of the same kind in Figs. 6(d) and 6(e); hence the dynamic Chern numbers are zero therein.

By contrast, when projected on the BZ, as shown in Figs. 6(f)-6(j), the spin textures feature no skyrmion structures, and the dynamics approach steady state in all $k$ sectors. 
[1] H. J. Carmichael, Quantum Trajectory Theory for Cascaded Open Systems, Phys. Rev. Lett. 70, 2273 (1993).

[2] C. M. Bender, Making sense of non-Hermitian Hamiltonians, Rep. Prog. Phys. 70, 947 (2007).

[3] V. V. Konotop, J. Yang, and D. A. Zezyulin, Nonlinear waves in $\mathcal{P} T$-symmetric systems, Rev. Mod. Phys. 88, 035002 (2016).

[4] R. El-Ganainy, K. G. Makris, M. Khajavikhan, Z. H. Musslimani, S. Rotter, and D. N. Christodoulides, NonHermitian physics and $\mathcal{P} T$ symmetry, Nat. Phys. 14, 11 (2018).

[5] C. M. Bender and S. Boettcher, Real Spectra in Non-Hermitian Hamiltonians Having $\mathcal{P} T$ Symmetry, Phys. Rev. Lett. 80, 5243 (1998).

[6] M. A. Miri and A. Alù, Exceptional points in optics and photonics, Science 363, eaar7709 (2019).

[7] Y. Ashida, Z. Gong, and M. Ueda, Non-Hermitian physics, arXiv:2006.01837.

[8] K. Kawabata, K. Shiozaki, M. Ueda, and M. Sato, Symmetry and Topology in Non-Hermitian Physics, Phys. Rev. X 9, 041015 (2019).

[9] H. Zhou and J. Y. Lee, Periodic table for topological bands with non-Hermitian symmetries, Phys. Rev. B 99, 235112 (2019).

[10] A. Ghatak and T. Das, New topological invariants in nonHermitian systems, J. Phys.: Condens. Matter 31, 263001 (2019).

[11] S. Yao and Z. Wang, Edge States and Topological Invariants of Non-Hermitian Systems, Phys. Rev. Lett. 121, 086803 (2018).

[12] S. Yao, F. Song, and Z. Wang, Non-Hermitian Chern Bands, Phys. Rev. Lett. 121, 136802 (2018).

[13] X.-Z. Zhang and J.-B. Gong, Non-Hermitian Floquet topological phases: Exceptional points, coalescent edge modes, and the skin effect, Phys. Rev. B 101, 045415 (2020).

[14] K. Yokomizo and S. Murakami, Non-Bloch Band Theory of Non-Hermitian Systems, Phys. Rev. Lett. 123, 066404 (2019).

[15] C. H. Lee and R. Thomale, Anatomy of skin modes and topology in non-Hermitian systems, Phys. Rev. B 99, 201103(R) (2019).

[16] F. K. Kunst, E. Edvardsson, J. C. Budich, and E. J. Bergholtz, Biorthogonal Bulk-Boundary Correspondence in Non-Hermitian Systems, Phys. Rev. Lett. 121, 026808 (2018).

[17] D. S. Borgnia, A. J. Kruchkov, and R.-J. Slager, Non-Hermitian Boundary Modes and Topology, Phys. Rev. Lett. 124, 056802 (2020).

[18] X.-R. Wang, C.-X. Guo, and S.-P. Kou, Defective edge states and number-anomalous bulk-boundary correspondence in nonHermitian topological systems, Phys. Rev. B 101, 121116(R) (2020).

[19] A. McDonald, T. Pereg-Barnea, and A. A. Clerk, PhaseDependent Chiral Transport and Effective Non-Hermitian Dynamics in a Bosonic Kitaev-Majorana Chain, Phys. Rev. X 8, 041031 (2018).

[20] V. M. Martinez Alvarez, J. E. Barrios Vargas, and L. E. F. Foa Torres, Non-Hermitian robust edge states in one dimension: Anomalous localization and eigenspace condensation at exceptional points, Phys. Rev. B 97, 121401(R) (2018).

[21] K. Zhang, Z. Yang, and C. Fang, Correspondence between Winding Numbers and Skin Modes in Non-Hermitian Systems, Phys. Rev. Lett. 125, 126402 (2020).

[22] N. Okuma, K. Kawabata, K. Shiozaki, and M. Sato, Topological Origin of Non-Hermitian Skin Effects, Phys. Rev. Lett. 124, 086801 (2020).
[23] Z.-S. Yang, K. Zhang, C. Fang, and J.-P. Hu, NonHermitian Bulk-Boundary Correspondence and Auxiliary Generalized Brillouin Zone Theory, Phys. Rev. Lett. 125, 226402 (2020).

[24] T. E. Lee, Anomalous Edge State in a Non-Hermitian Lattice, Phys. Rev. Lett. 116, 133903 (2016).

[25] T.-S. Deng and W. Yi, Non-Bloch topological invariants in a non-Hermitian domain wall system, Phys. Rev. B 100, 035102 (2019).

[26] T. Li, Y.-S. Zhang, and W. Yi, Two-dimensional quantum walk with non-Hermitian skin effects, Chin. Phys. Lett. 38, 030301 (2021).

[27] T. Helbig, T. Hofmann, S. Imhof, M. Abdelghany, T. Kliessling, L. W. Molenkamp, C. H. Lee, A. Szameit, M. Greiter, and R. Thomale, Generalized bulk-boundary correspondence in nonHermitian topolectrical circuits, Nat. Phys. 16, 747 (2020).

[28] L. Xiao, T.-S. Deng, K. Wang, G. Zhu, Z. Wang, W. Yi, and P. Xue, Observation of non-Hermitian bulk-boundary correspondence in quantum dynamics, Nat. Phys. 16, 761 (2020).

[29] A. Ghatak, M. Brandenbourger, J. van Wezel, and C. Coulais, Observation of non-Hermitian topology and its bulk-edge correspondence in an active mechanical metamaterial, Proc. Natl. Acad. Sci. USA 117, 29561 (2020).

[30] T. Hofmann, T. Helbig, F. Schindler, N. Salgo, M. Brzezińska, M. Greiter, T. Kiessling, D. Wolf, A. Vollhardt, A. Kabaši, C. H. Lee, A. Bilušić, R. Thomale, and T. Neupert, Reciprocal skin effect and its realization in a topolectrical circuit, Phys. Rev. Res. 2, 023265 (2020).

[31] S. Weidemann, M. Kremer, T. Helbig, T. Hofmann, A. Stegmaier, M. Greiter, R. Thomale, and A. Szameit, Topological funneling of light, Science 368, 311 (2020).

[32] L. Xiao, T.-S. Deng, K.-K. Wang, Z. Wang, W. Yi, and P. Xue, Observation of non-Bloch parity-time symmetry and exceptional points, arXiv:2009.07288.

[33] C. Yang, L. Li, and S. Chen, Dynamical topological invariant after a quantum quench, Phys. Rev. B 97, 060304(R) (2018).

[34] Z. Gong and M. Ueda, Topological Entanglement-Spectrum Crossing in Quench Dynamics, Phys. Rev. Lett. 121, 250601 (2018).

[35] X. Qiu, T.-S. Deng, Y. Hu, P. Xue, and W. Yi, Fixed points and dynamic topological phenomena in a parity-time-symmetric quantum quench, iScience 20, 392 (2019).

[36] J. Eisert, M. Friesdorf, and C. Gogolin, Quantum many-body systems out of equilibrium, Nat. Phys. 11, 124 (2015).

[37] M. D. Caio, N. R. Cooper, and M. J. Bhaseen, Quantum Quenches in Chern Insulators, Phys. Rev. Lett. 115, 236403 (2015).

[38] S. Vajna and B. Dóra, Topological classification of dynamical phase transitions, Phys. Rev. B 91, 155127 (2015).

[39] J. C. Budich and M. Heyl, Dynamical topological order parameters far from equilibrium, Phys. Rev. B 93, 085416 (2016).

[40] Z. Huang and A. V. Balatsky, Dynamical Quantum Phase Transitions: Role of Topological Nodes in Wave Function Overlaps, Phys. Rev. Lett. 117, 086802 (2016).

[41] Y. Hu, P. Zoller, and J. C. Budich, Dynamical Buildup of a Quantized Hall Response from Nontopological States, Phys. Rev. Lett. 117, 126803 (2016).

[42] J. H. Wilson, J. C. W. Song, and G. Refael, Remnant Geometric Hall Response in a Quantum Quench, Phys. Rev. Lett. 117, 235302 (2016). 
[43] M. D. Caio, N. R. Cooper, and M. J. Bhaseen, Hall response and edge current dynamics in Chern insulators out of equilibrium, Phys. Rev. B 94, 155104 (2016).

[44] C. Wang, P. Zhang, X. Chen, J. Yu, and H. Zhai, Scheme to Measure the Topological Number of a Chern Insulator from Quench Dynamics, Phys. Rev. Lett. 118, 185701 (2017).

[45] L. Zhang, L. Zhang, S. Niu, and X.-J. Liu, Dynamical classification of topological quantum phases, Sci. Bull. 63, 1385 (2018).

[46] M. Heyl, Dynamical quantum phase transitions: A review, Rep. Prog. Phys. 81, 054001 (2018).

[47] L. Zhou, Dynamical characterization of non-Hermitian Floquet topological phases in one dimension, Phys. Rev. B 100, 184314 (2019).

[48] J. Yu, Measuring Hopf links and Hopf invariants in a quenched topological Raman lattice, Phys. Rev. A 99, 043619 (2019).

[49] S. Sayyad, J. Yu, A. G. Grushin, and L. M. Sieberer, Entanglement spectrum crossings reveal non-Hermitian dynamical topology, arXiv:2011.10601.

[50] N. Fläschner, D. Vogel, M. Tarnowski, B. S. Rem, D.-S. Lühmann, M. Heyl, J. C. Budich, L. Mathey, K. Sengstock, and C. Weitenberg, Observation of dynamical vortices after quenches in a system with topology, Nat. Phys. 14, 265 (2018).

[51] M. Tarnowski, F. Nur Ünal, N. Fläschner, B. S. Rem, A. Eckardt, K. Sengstock, and C. Weitenberg, Measuring topology from dynamics by obtaining the Chern number from a linking number, Nat. Commun. 10, 1728 (2019).

[52] W. Sun, C.-R. Yi, B.-Z. Wang, W.-W. Zhang, B. C. Sanders, X.-T. Xu, Z.-Y. Wang, J. Schmiedmayer, Y. Deng, X.-J. Liu, S. Chen, and J.-W. Pan, Uncover Topology by Quantum Quench Dynamics, Phys. Rev. Lett. 121, 250403 (2018).

[53] K. Wang, X. Qiu, L. Xiao, X. Zhan, Z. Bian, W. Yi, and P. Xue, Simulating Dynamic Quantum Phase Transitions in Photonic Quantum Walks, Phys. Rev. Lett. 122, 020501 (2019).

[54] F. N. Ünal, A. Bouhon, and R.-J. Slager, Topological Euler Class as a Dynamical Observable in Optical Lattices, Phys. Rev. Lett. 125, 053601 (2020).

[55] K. Wang, X. Qiu, L. Xiao, X. Zhan, Z. Bian, B. C. Sanders, W. Yi, and P. Xue, Observation of emergent momentum-time skyrmions in parity-time-symmetric non-unitary quench dynamics, Nat. Commun. 10, 2293 (2019).

[56] S. Longhi, Probing non-Hermitian skin effect and non-Bloch phase transitions, Phys. Rev. Res. 1, 023013 (2019).

[57] S. Longhi, Non-Bloch $\mathcal{P} T$-symmetry breaking in nonHermitian photonic quantum walks, Opt. Lett. 44, 5804 (2019).

[58] W. P. Su, J. R. Schrieffer, and A. J. Heeger, Solitons in Polyacetylene, Phys. Rev. Lett. 42, 1698 (1979).

[59] See the appendices for details.

[60] D. C. Brody, Biorthogonal quantum mechanics, J. Phys. A: Math. Theor. 47, 035305 (2014). 